TRANSACTIONS OF THE

AMERICAN MATHEMATICAL SOCIETY

Volume 349, Number 5, May 1997, Pages 1901-1918

S 0002-9947(97)01903-X

\title{
ON THE EXTREMAL STRUCTURE OF THE UNIT BALLS OF BANACH SPACES OF WEAKLY CONTINUOUS FUNCTIONS AND THEIR DUALS
}

\author{
ZHIBAO HU AND MARK A. SMITH
}

\begin{abstract}
A sufficient and then a necessary condition are given for a function to be an extreme point of the unit ball of the Banach space $C(K,(X, w))$ of continuous functions, under the supremum norm, from a compact Hausdorff topological space $K$ into a Banach space $X$ equipped with its weak topology $w$. Strongly extreme points of the unit ball of $C(K,(X, w))$ are characterized as the norm-one functions that are uniformly strongly extreme point valued on a dense subset of $K$. It is shown that a variety of stronger types of extreme points (e.g. denting points) never exist in the unit ball of $C(K,(X, w))$. Lastly, some naturally arising and previously known extreme points of the unit ball of $C(K,(X, w))^{*}$ are shown to actually be strongly exposed points.
\end{abstract}

\section{INTRODUCTION}

Several types of extreme points of convex sets in Banach spaces have been invented and studied during the last six decades. For a specific Banach space, it is always of interest to discover whether the closed unit ball has any extreme points and, if so, to exactly describe them; it is of further interest to determine which, if any, of these extreme points are in fact one of the many stronger types of extreme points appearing in the literature. Such discoveries and determinations are the subject matter of this paper.

Let $K$ be an infinite compact Hausdorff topological space, let $X$ be a real Banach space, and let $w$ denote the weak topology on $X$. Let $C(K,(X, w))$ denote the Banach space of all continuous functions from $K$ into $(X, w)$ equipped with the supremum norm; the geometry of this Banach space has been the subject of several recent investigations (see [ADLR], [C], [DD], and [M]).

In this paper, the extremal structure of the closed unit ball of $C(K,(X, w))$ relative to the extremal structure of the closed unit ball of $X$ is investigated. The rather elusive extreme point structure of the unit ball of $C(K,(X, w))$ is discussed and it is compared to the extreme point structure situation in the unit ball of $C(K, X)$, the space of all continuous functions from $K$ into $X$ equipped with the supremum norm (here $X$ is considered with its norm topology). Next, the strongly extreme points of the unit ball of $C(K,(X, w))$ are characterized in a very natural manner; the strongly extreme point situation here is quite different from that in

Received by the editors November 9, 1995.

1991 Mathematics Subject Classification. Primary 46B20, 46E40.

Key words and phrases. Extreme points, strongly extreme points, points of continuity, denting points, spaces of weakly continuous functions.

The second author was supported in part by a Miami University Summer Research Grant. 
the unit ball of $C(K, X)$ from one point of view and is exactly the same from another point of view. Examples are provided that illustrate this difference, and a proposition is given that witnesses this sameness. Finally, it is shown that the unit ball of $C(K,(X, w))$ never has many of the stronger types of extreme points (for example, denting points).

Also, in this paper, the extremal structure of the unit ball of the dual space $C(K,(X, w))^{*}$ is examined. The lack of a concrete representation of this dual space (as opposed to the dual space $\left.C(K, X)^{*}\right)$ considerably clouds the extreme point situation. It is known, from [ADLR], that $C(K, X)^{*}$ is always complemented in $C(K,(X, w))^{*}$; hence the extreme points of the unit ball of $C(K, X)^{*}$, which are well known, have a chance to also be extreme points in $C(K,(X, w))^{*}$. Several examples of classical Banach spaces are given for which this is the case. The main results here are strengthenings of a theorem of P-K. Lin $[\mathrm{L}]$, as illuminated by M. Cambern $[\mathrm{C}]$; these are strengthenings that identify, for the first time, some denting points and some strongly exposed points of the unit ball of $C(K,(X, w))^{*}$.

The authors wish to express considerable thanks to Professor P.N. "Paddy" Dowling for many helpful conversations regarding the subject matter of this paper.

\section{Definitions And Preliminaries}

For a real Banach space $E$, let $B_{E}$ denote the closed unit ball of $E$ and let $S_{E}$ denote the unit sphere of $E$. The unit ball $B_{E}$ is said to be stable provided the mapping from $B_{E} \times B_{E}$ into $B_{E}$ that sends $(x, y)$ to $\frac{1}{2}(x+y)$ is an open mapping. The dual space of a Banach space $E$ is denoted by $E^{*}$ and the value of $x^{*}$ in $E^{*}$ at $x$ in $E$ is denoted by $\left\langle x^{*}, x\right\rangle$. The notation $\|x \pm z\| \leq 1+\delta$ means " $\|x+z\| \leq 1+\delta$ and $\|x-z\| \leq 1+\delta$."

The following is a well-known list (included here for completeness sake) of geometrical notions.

(i) A point $x$ in $B_{E}$ is called an extreme point of $B_{E}$ provided $x$ is not the midpoint of any non-trivial line segment lying in $B_{E}$.

(ii) A point $x$ in $B_{E}$ is called a strongly extreme point of $B_{E}$ provided that for every $\varepsilon>0$ there exists a $\delta>0$ such that $\|x \pm z\| \leq 1+\delta$ for $z$ in $E$ implies $\|z\| \leq \varepsilon$.

(iii) A point $x$ in $B_{E}$ is called a point of continuity of $B_{E}$ provided that whenever $\left\{x_{\lambda}\right\}_{\lambda \in \Lambda}$ is a net in $B_{E}$ which converges weakly to $x$, it follows that $\left\{x_{\lambda}\right\}_{\lambda \in \Lambda}$ converges in norm to $x$. If nets are replaced by sequences in this condition, then $x$ is called a point of sequential continuity of $B_{E}$.

(iv) A point $x$ in $B_{E}$ is called a denting point of $B_{E}$ provided $x$ is not an element of the closed convex hull of $\left\{y \in B_{E}:\|y-x\|>\varepsilon\right\}$ for each $\varepsilon>0$.

(v) A point $x$ in $B_{E}$ is called a strongly exposed point of $B_{E}$ provided that there exists $x^{*}$ in $B_{E^{*}}$ such that $\left\langle x^{*}, x\right\rangle=\left\|x^{*}\right\|=\|x\|=1$ and whenever $\left\{x_{n}\right\}_{n=1}^{\infty}$ is a sequence in $B_{E}$ such that $\lim _{n \rightarrow \infty}\left\langle x^{*}, x_{n}\right\rangle=1$, it follows that

$$
\lim _{n \rightarrow \infty}\left\|x-x_{n}\right\|=0
$$

in this case, the functional $x^{*}$ is said to strongly expose $B_{E}$ at $x$.

It is known, from [LLT], that a point in $B_{E}$ is a denting point of $B_{E}$ if and only if it is both a point of continuity and an extreme point of $B_{E}$. For $x^{*}$ in $E^{*}$ and 
$\delta>0$, the slice of $B_{E}$ determined by $x^{*}$ and $\delta$ is the set

$$
S\left(x^{*}, B_{E}, \delta\right)=\left\{y \in B_{E}:\left\langle x^{*}, y\right\rangle \geq\left\|x^{*}\right\|-\delta\right\} .
$$

It is straightforward to show that $x$ is a denting point of $B_{E}$ if and only if for every $\varepsilon>0$ there exist $x^{*}$ in $E^{*}$ and $\delta>0$ such that $\operatorname{diam} S\left(x^{*}, B_{E}, \delta\right)<\varepsilon$. Also $x$ is a strongly exposed point of $B_{E}$ if and only if there exists an element $x^{*}$ in $S_{E^{*}}$ such that $\left\langle x^{*}, x\right\rangle=1$ and $\lim _{\delta \rightarrow 0^{+}} \operatorname{diam} S\left(x^{*}, B_{E}, \delta\right)=0$.

If $E$ is a dual space, the notions of a weak $k^{*}$ point of continuity and a weak* point of sequential continuity of $B_{E}$ are defined as in (iii) above replacing weak convergence by weak* convergence; the notion of a weak ${ }^{*}$ denting point of $B_{E}$ is defined as in (iv) above replacing the closed convex hull by the weak ${ }^{*}$ closed convex hull of the set given there; and the notion of a weak ${ }^{*}$ strongly exposed point of $B_{E}$ is defined as in (v) above insisting that the strongly exposing functional belongs to the predual of $E$. Statements concerning weak ${ }^{*}$ denting points and weak* strongly exposed points can be made which correspond to the statements made immediately after (v) above about denting points and strongly exposed points.

With the obvious abbreviations, the diagram below gives the relative strengths of all these notions.

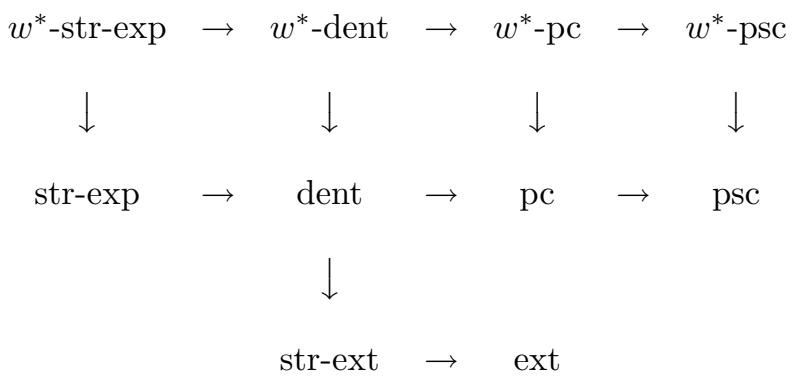

The dual space $C(K, X)^{*}$ can be identified with the space of all countably additive, $X^{*}$-valued, Borel measures of bounded variation on $K$ equipped with the variation norm, where the action of $\mu$ in $C(K, X)^{*}$ on $f$ in $C(K, X)$ is given by $\langle\mu, f\rangle=$ $\int_{K} f d \mu$. The space $C(K, X)$ may be considered as a closed subspace of $C(K,(X, w))$. For $f$ in $C(K,(X, w))$, define $\tilde{f}: K \times B_{X^{*}} \rightarrow \mathbb{R}$ by

$$
\tilde{f}\left(t, x^{*}\right)=\left\langle x^{*}, f(t)\right\rangle \text { for }\left(t, x^{*}\right) \text { in } K \times B_{X^{*}} .
$$

Then $\tilde{f}$ is a continuous function on the completely regular topological space $K \times$ $B_{X^{*}}$; here $B_{X^{*}}$ is equipped with its norm topology, and

$$
\sup \left\{\left|\tilde{f}\left(t, x^{*}\right)\right|:\left(t, x^{*}\right) \in K \times B_{X^{*}}\right\}=\|f\| .
$$

Each $\tilde{f}$ has a unique continuous extension to a function $\bar{f}$ defined on the Stone-Cech compactification $Y$ of $K \times B_{X^{*}}$. Thus $C(K,(X, w))$ may be considered as a closed subspace of $C(Y)=C(Y, \mathbb{R})$ via the mapping $f \longmapsto \bar{f}$. For $g$ in $C(K)$ and $x$ in $X$, the symbol $g \otimes x$ denotes the mapping from $K \times B_{X^{*}}$ into $\mathbb{R}$ given by

$$
(g \otimes x)\left(t, x^{*}\right)=g(t)\left\langle x^{*}, x\right\rangle \text { for }\left(t, x^{*}\right) \text { in } K \times B_{X^{*}}
$$

and $\overline{g \otimes x}$ denotes the continuous extension of $g \otimes x$ to $Y$.

It should be noted that, in the verification of an example or in the proof of a theorem, consecutive claims and displays are numbered with those numbers referring only to the argument within that verification or proof. 


\section{The SPace $C(K,(X, w))$}

The first result in this section gives a sufficient condition and a necessary condition for an element of $C(K,(X, w))$ to be an extreme point of the unit ball of $C(K,(X, w))$. The proof of assertion (i) is straightforward, and assertion (ii) follows from the fact that, for every element $f$ in $C(K,(X, w))$, the set $\{t \in K: f$ is norm continuous at $t\}$ is a dense $G_{\delta}$ subset of $K$ (see the proof of Theorem 5 below).

Proposition 1. Let $f$ be an element of $C(K,(X, w))$.

(i) If the set of all $t$ in $K$ for which $f(t)$ is an extreme point of $B_{X}$ is dense in $K$, then $f$ is an extreme point of $B_{C(K,(X, w))}$.

(ii) If $f$ is an extreme point of $B_{C(K,(X, w))}$, then $\|f(t)\|=1$ for all $t$ in a dense $G_{\delta}$ subset of $K$ and $f(t)$ is an extreme point of $B_{X}$ for all isolated points $t$ in $K$.

Recall, from $[\mathrm{BLP}]$, that there is a Banach space $X$ with $\operatorname{dim} X=4$ and an extreme point $f$ of $B_{C([0,1], X)}$ such that there is no $t$ in $[0,1]$ for which $f(t)$ is an extreme point of $B_{X}$. The existence of this example shows that, in general, assertion (ii) of Proposition 1 is as strong as possible and that the converse of assertion (i) is certainly not true. However, if $X$ is strictly convex (that is, every element of $S_{X}$ is an extreme point of $\left.B_{X}\right)$, then the extreme points of $B_{C(K,(X, w))}$ can be nicely characterized.

Corollary 2. If $X$ is a strictly convex Banach space, then $f$ is an extreme point of $B_{C(K,(X, w))}$ if and only if the set of all $t$ in $K$ for which $f(t)$ is an extreme point of $B_{X}$ is a dense subset of $K$.

Recall (see [G] or [DHS]) that if $B_{X}$ is stable, then a function $f$ in $C(K, X)$ is an extreme point of $B_{C(K, X)}$ if and only if $f(t)$ is an extreme point of $B_{X}$ for all $t$ in $K$. The corresponding statement for $f$ in $C(K,(X, w))$ is false, as the following example shows.

Example 3. Let $K=\left\{\frac{1}{n}: n \in \mathbb{N}\right\} \cup\{0\}$ with the topology inherited from $\mathbb{R}$ and let $X=\ell^{2}$ with its usual norm. Define $f: K \rightarrow X$ by $f\left(\frac{1}{n}\right)=e_{n}$ for each $n$ in $\mathbb{N}$, where $\left\{e_{n}\right\}_{n=1}^{\infty}$ is the usual unit vector basis for $\ell^{2}$, and $f(0)=0$. Then $f$ is in $C(K,(X, w))$ and is an extreme point of $B_{C(K,(X, w))}$ by Proposition 1 . Yet $f$ is not everywhere extreme point valued, since $f(0)=0$, even though $B_{X}$ is stable.

The function $f$ in Example 3 is not particularly surprising; it exists because the set of extreme points of $B_{\ell^{2}}$ is not weakly closed. Note that in a Banach space $X$ for which $B_{X}$ is stable, the set of extreme points of $B_{X}$ is norm closed. Since $B_{X}$ is stable whenever $X$ is strictly convex, it is natural to ask whether the statement of Corollary 2 remains true whenever the hypothesis that $X$ is strictly convex is replaced by the hypothesis that $B_{X}$ is stable. The authors have no counterexample, but there seems to be little immediate evidence to support an affirmative conjecture.

Definition 4. A subset $A$ of $B_{X}$ is said to be uniformly strongly extreme provided for every $\varepsilon>0$ there exists $\delta>0$ such that, for all $x$ in $A$, if $\|x \pm z\| \leq 1+\delta$ for $z$ in $X$, then $\|z\| \leq \varepsilon$.

The notion of uniformly strongly extreme subset of $B_{X}$ permits the very natural and complete characterization of the strongly extreme points of $B_{C(K,(X, w))}$ given 
in the next theorem. From one point of view, the strongly extreme point situation in $C(K,(X, w))$ is quite different from that in $C(K, X)$, but, from another point of view, it is exactly the same. The difference is illustrated in the examples following the theorem, and the sameness is given in the proposition following the examples. Note that Theorem 5 can be read with or without the parenthetical "and $G_{\delta}$ "; thus it is actually the statement of the equivalence of three assertions.

Theorem 5. An element $f$ of $C(K,(X, w))$ is a strongly extreme point of $B_{C(K,(X, w))}$ if and only if there exists a dense (and $G_{\delta}$ ) subset $D$ of $K$ such that $f(D)$ is a uniformly strongly extreme subset of $B_{X}$.

Proof. Let $f$ be in $C(K,(X, w))$ and suppose there exists a dense subset $D$ of $K$ such that $f(D)$ is a uniformly strongly extreme subset of $B_{X}$. Since $f$ is weakly continuous, since $D$ is dense and since $B_{X}$ is weakly closed, it follows that $\|f(t)\|$ $\leq 1$ for all $t$ in $K$; that is, $\|f\| \leq 1$. Now, let $\varepsilon>0$ be given and use the hypothesis on $f(D)$ to produce a corresponding $\delta>0$. If $h$ is in $C(K,(X, w))$ and is such that $\|f \pm h\| \leq 1+\delta$, then

$$
\|f(t) \pm h(t)\| \leq 1+\delta \text { for all } t \text { in } D,
$$

and hence $\|h(t)\| \leq \varepsilon$ for all $t$ in $D$. As above, it follows that $\|h\| \leq \varepsilon$. This shows that $f$ is a strongly extreme point of $B_{C(K,(X, w))}$.

Conversely, suppose $f$ is a strongly extreme point of $B_{C(K,(X, w))}$. Since $f$ is in $C(K,(X, w))$, it follows that $f(K)$ is weakly compact in $X$ and hence $f(K)$ has the point-of-continuity property. For each $n$ in $\mathbb{N}$, let

$O_{n}=\{t \in K:$ there exists an open set $V$ containing $t$ such that diam $\left.f(V)<\frac{1}{n}\right\}$.

Note that, by definition, each $O_{n}$ is open in $K$ and

$$
\bigcap_{n=1}^{\infty} O_{n}=\{t \in K: f \text { is norm continuous at } t\} .
$$

Claim 1. The set $O_{n}$ is dense in $K$ for each $n$ in $\mathbb{N}$.

To establish this claim, let $V$ be a nonempty, open subset of $K$ and fix $n$ in $\mathbb{N}$. Since $f(V) \subseteq f(K)$ and $f(K)$ has the point-of-continuity property, there exists a weakly open, nonempty set $U$ in $X$ with $U \cap f(V)$ nonempty and $\operatorname{diam}(U \cap f(V))$ $<\frac{1}{n}$. Let $W=f^{-1}(U) \cap V$. Then $W$ is nonempty, open and a subset of $V \cap O_{n}$. This establishes the claim.

Claim 2. For every $\varepsilon>0$, there exists $\delta>0$ such that the set

$$
K_{\varepsilon, \delta}=\{t \in K:\|f(t) \pm z\| \leq 1+\delta \text { implies }\|z\| \leq \varepsilon\}
$$

is dense in $K$.

To establish this claim, suppose otherwise. Then there exists $\varepsilon>0$ such that for all $\delta>0$ it is the case that $\bar{K}_{\varepsilon, \delta} \neq K$. Let $G_{n}=K \backslash \bar{K}_{\varepsilon, \frac{1}{n}}$ for each $n$ in $\mathbb{N}$. Then $G_{n}$ is nonempty and open, and $G_{n} \supseteq G_{n+1}$ for each $n$ in $\mathbb{N}$. Consider the following two alternatives:

Case 1. There exists $m$ in $\mathbb{N}$ such that $G_{n} \subseteq G_{m} \subseteq \bar{G}_{n}$ for each $n \geq m$. 
In this case, note that since, by Claim 1, the set $O_{n} \cap G_{m}$ is open and dense in $G_{m}$ for each $n$ in $\mathbb{N}$, since $G_{n}$ is open and dense in $G_{m}$ for each $n \geq m$, and since $G_{m}$ is an open (and hence Baire) subset of $K$, it follows that

$$
\left(\bigcap_{n=1}^{\infty} O_{n}\right) \cap\left(\bigcap_{n=m}^{\infty} G_{n}\right) \cap G_{m} \quad \text { is dense in } G_{m} .
$$

Let $t_{0}$ be a fixed element of this dense subset of $G_{m}$. Then $f$ is norm continuous at $t_{0}$, since $t_{0}$ is in $\bigcap_{n=1}^{\infty} O_{n}$, and $f\left(t_{0}\right)$ is not a strongly extreme point of $B_{X}$, since $t_{0}$ is in $\bigcap_{n=m}^{\infty} G_{n}$. Now, proceed as in the proof of Theorem 2 of [DHS] to produce a sequence $\left\{h_{n}\right\}_{n=1}^{\infty}$ in $C(K, X)$, and hence in $C(K,(X, w))$, such that $\left\|f \pm h_{n}\right\| \leq 1+\frac{2}{n}$ and $\left\|h_{n}\right\| \geq \varepsilon$ for each $n$ in $\mathbb{N}$. This shows that $f$ is not a strongly extreme point of $B_{C(K,(X, w))}$, contrary to the hypothesis.

Case 2. For every $m$ in $\mathbb{N}$, there exists $n>m$ such that $G_{n} \subseteq G_{m} \nsubseteq \bar{G}_{n}$.

In this case, there exists an increasing sequence $\left\{m_{n}\right\}_{n=1}^{\infty}$ in $\mathbb{N}$ such that

$$
A_{n}=G_{m_{n}} \backslash \bar{G}_{m_{n+1}} \text { is nonempty for each } n \text { in } \mathbb{N} \text {. }
$$

Note that $\left\{A_{n}\right\}_{n=1}^{\infty}$ is a pairwise disjoint sequence of nonempty, open subsets of $K$. For each $n$ in $\mathbb{N}$, choose $t_{n}$ in $A_{n}$ such that $f$ is norm continuous at $t_{n}$; this can be done (see Case 1 above) since $A_{n}$ is open and hence $A_{n} \cap\left(\bigcap_{i=1}^{\infty} O_{i}\right)$ is dense in $A_{n}$. To show that this circumstance implies that $f$ is not a strongly extreme point of $B_{C(K,(X, w))}$, which is contrary to the hypothesis, let $\eta>0$ be given. Choose and fix $n$ in $\mathbb{N}$ such that $m_{n}>\frac{2}{\eta}$. Since $f$ is norm continuous at $t_{n}$, there exists an open subset $U$ containing $t_{n}$ such that

$$
U \subseteq A_{n} \text { and } \operatorname{diam} f(U)<\frac{\eta}{2} .
$$

Now, there exists a continuous function $g: K \rightarrow[0,1] \operatorname{such}$ that $\operatorname{supp}(g) \subseteq$ $U$ and $g\left(t_{n}\right)=1$. Since $t_{n}$ is in $A_{n}$, which is a subset of $G_{m_{n}}$, it follows that there exists $z$ in $X$ such that

$$
\left\|f\left(t_{n}\right) \pm z\right\| \leq 1+\frac{1}{m_{n}} \text { and }\|z\|>\varepsilon .
$$

Define $h: K \rightarrow X$ by $h(t)=g(t) z$ for $t$ in $K$. Then $h$ is in $C(K,(X, w))$ and $\|h\| \geq\left\|h\left(t_{n}\right)\right\|=\|z\|>\varepsilon$. For $t$ in $K \backslash U$,

$$
\|f(t) \pm h(t)\|=\|f(t)\| \leq 1
$$

while, for $t$ in $U$,

$$
\begin{aligned}
\|f(t) \pm h(t)\| & \leq\left\|f(t)-f\left(t_{n}\right)\right\|+\left\|f\left(t_{n}\right) \pm g(t) z\right\| \\
& <\frac{\eta}{2}+1+\frac{1}{m_{n}} \\
& <1+\eta .
\end{aligned}
$$

Thus $\|f \pm h\| \leq 1+\eta$ and $\|h\| \geq \varepsilon$. This establishes the claim.

For every $n$ in $\mathbb{N}$, by Claim 2, there exists $i_{n}$ in $\mathbb{N}$ such that $K_{\frac{1}{n}, \frac{1}{i_{n}}}$ is dense in $K$. For ease of notation, write $K_{\left(n, i_{n}\right)}$ for this dense set.

Claim 3. The set $O_{2 i_{n}} \subseteq K_{\left(n, 2 i_{n}\right)}$ for each $n$ in $\mathbb{N}$. 
To establish this claim, fix $n$ in $\mathbb{N}$ and let $t$ be in $O_{2 i_{n}}$. Then there exists an open set $V$ containing $t$ such that $\operatorname{diam} f(V)<\frac{1}{2 i_{n}}$. Since $K_{\left(n, i_{n}\right)}$ is dense in $K$, there exists a point $k$ in $K_{\left(n, i_{n}\right)} \cap V$. Now, if $z$ is in $X$ such that $\|f(t) \pm z\| \leq 1+\frac{1}{2 i_{n}}$, then

$$
\begin{aligned}
\|f(k) \pm z\| & \leq\|f(k)-f(t)\|+\|f(t) \pm z\| \\
& <\frac{1}{2 i_{n}}+1+\frac{1}{2 i_{n}} \\
& =1+\frac{1}{i_{n}}
\end{aligned}
$$

and hence $\|z\| \leq \frac{1}{n}$, since $k$ is in $K_{\left(n, i_{n}\right)}$. This shows $t$ is in $K_{\left(n, 2 i_{n}\right)}$ and the claim is established.

Finally, let $D=\bigcap_{n=1}^{\infty} O_{2 i_{n}}$. Then $D$ is a $G_{\delta}$ subset of $K$ and $D$ is dense in $K$, since each $O_{2 i_{n}}$ is open and dense in $K$. Also, by Claim 3,

$$
D \subseteq \bigcap_{n=1}^{\infty} K_{\left(n, 2 i_{n}\right)}
$$

which yields that $f(D)$ is a uniformly strongly extreme subset of $B_{X}$. This completes the proof of the theorem.

The next two examples show that each of the following situations can occur:

(i) there exist an infinite compact Hausdorff space $K$, a real Banach space $X$ and a strongly extreme point $f$ in $B_{C(K,(X, w))}$ such that not every value of $f$ is a strongly extreme point of $B_{X}$; and

(ii) there exist an infinite compact Hausdorff space $K$, a real Banach space $X$ and $f$ in $B_{C(K,(X, w))}$ such that $f(t)$ is a strongly extreme point of $B_{X}$ for all $t$ in $K$ yet $f$ is not a strongly extreme point of $B_{C(K,(X, w))}$.

These examples in $C(K,(X, w))$ should be compared to the situation in $C(K, X)$; recall, from [DHS], that $f$ in $C(K, X)$ is a strongly extreme point of $B_{C(K, X)}$ if and only if $f(t)$ is a strongly extreme point of $B_{X}$ for every $t$ in $K$.

Example 6. (This is really Example 3 revisited.) Let the topological space $K$, the Banach space $X$ and the function $f$ be those of Example 3. Then $f$ is a strongly extreme point of $B_{C(K,(X, w))}$, by Theorem 5 , since $D=\left\{\frac{1}{n}: n \in \mathbb{N}\right\}$ is dense in $K$ and $f(D)=\left\{e_{n}: n \in \mathbb{N}\right\}$ is a uniformly strongly extreme subset of $B_{X}$ (this is true since $X$ is uniformly rotund). Yet $f$ is not everywhere strongly extreme point valued since $f(0)=0$.

Example 7. For each $i$ in $\mathbb{N}$, let

$$
B_{i}=c o\left\{( \pm 1,0),(0, \pm 1),\left( \pm \frac{i}{i+1}, \pm \frac{i}{i+1}\right)\right\}
$$

the "octagon" ball in $\mathbb{R}^{2}$, and let $|\cdot|_{i}$ be the Minkowski functional of $B_{i}$. Then let $X_{i}=\mathbb{R}^{3}$ with the norm $\|\cdot \cdot\| \|_{i}$ given by

$$
\|(\alpha, \beta, \gamma)\| \|_{i}=\left(|\alpha|^{2}+|(\beta, \gamma)|_{i}^{2}\right)^{\frac{1}{2}}
$$

that is, $X_{i}=\mathbb{R} \oplus_{2}\left(\mathbb{R}^{2},|\cdot|_{i}\right)$ for each $i$ in $\mathbb{N}$. Finally, let $X=\ell^{\infty}\left(X_{i}\right)$, the Banach sequence space, equipped with the usual supremum norm. Now, let

$$
e_{0}=(1,0,0) \text { and } e_{1}=(0,1,0)
$$


in $X_{i}$ and then let

$$
x_{0}=\left(e_{0}, e_{0}, e_{0}, \ldots\right)
$$

and

$$
x_{n}=(\underbrace{e_{0}, e_{0}, \ldots, e_{0}}_{n-1}, e_{1}, e_{0}, e_{0}, \ldots)
$$

for each $n$ in $\mathbb{N}$. Note $\left\{x_{n}\right\}_{n=1}^{\infty}$ and $x_{0}$ are in $X$ and $\left\|x_{n}\right\|=\left\|x_{0}\right\|=1$.

Claim 1. Each $x_{n}$ and $x_{0}$ are strongly extreme points of $B_{X}$.

To establish this claim, let $|\cdot|_{\infty}$ denote the supremum norm on $\mathbb{R}^{2}$ and then let $X_{0}=\mathbb{R}^{3}$ with the norm ||$|\cdot|||$ given by

$$
\|||(\alpha, \beta, \gamma) \mid\|=\left(|\alpha|^{2}+|(\beta, \gamma)|_{\infty}^{2}\right)^{\frac{1}{2}}
$$

that is, $X_{0}=\mathbb{R} \oplus_{2}\left(\mathbb{R}^{2},|\cdot|_{\infty}\right)$. Since $B_{X_{i}} \subseteq B_{X_{0}}$ and $B_{\left(\mathbb{R}^{2},|\cdot|_{\infty}\right)} \subseteq 2 B_{\left(\mathbb{R}^{2},|\cdot|_{i}\right)}$ for each $i$ in $\mathbb{N}$, it follows that

$$
\|z\| \leq\|z\|\left\|_{i} \leq 2|\|z\||\right.
$$

for all $z$ in $\mathbb{R}^{3}$. It is easily seen that $e_{0}$ is a strongly extreme point of $B_{X_{0}}$; in fact, $e_{0}$ is a strongly exposed point of $B_{X_{0}}$ exposed by the functional $(1,0,0)$ in $X_{0}^{*}$. Using this fact combined with (1), it follows that for every $\varepsilon>0$ there exists $\delta>0$ such that, for all $i$ in $\mathbb{N}$,

$$
\left\|\mid e_{0} \pm z\right\| \|_{i} \leq 1+\delta \text { implies }\|\mid z\|_{i} \leq 2 \varepsilon
$$

for all $z$ in $\mathbb{R}^{3}$. To show $x_{0}$ is a strongly extreme point of $B_{X}$, let $\varepsilon>0$ be given and suppose $y=(y(1), y(2), \ldots)$ is in $X$ such that $\left\|x_{0} \pm y\right\| \leq 1+\delta$, where $\delta$ is given as in (2). Then $\left\|e_{0}+y(i)\right\|_{i} \leq 1+\delta$ and hence $\|y(i)\| \|_{i} \leq 2 \varepsilon$ for each $i$ in $\mathbb{N}$. Thus $\|y\| \leq 2 \varepsilon$. This shows $x_{0}$ is a strongly extreme point of $B_{X}$. Now, fix $n$ in $\mathbb{N}$; to show $x_{n}$ is a strongly extreme point of $B_{X}$, let $\varepsilon>0$ be given. Consider the unit ball $B_{X_{n}}$ and note that $e_{1}$ is a strongly extreme point of $B_{X_{n}}$; in fact, $e_{1}$ is a strongly exposed point of $B_{X_{n}}$ exposed by the functional $(0,1,0)$ in $X_{n}^{*}$. Use this fact in combination with (2) to produce $\delta>0$ such that

$$
\left\|e_{1} \pm w\right\|_{n} \leq 1+\delta \text { implies }\|\mid w\|_{n} \leq \varepsilon
$$

for all $w$ in $\mathbb{R}^{3}$ and, for all $i$ in $\mathbb{N}$,

$$
\left\|e_{0} \pm z\right\| \|_{i} \leq 1+\delta \text { implies }\|z\|_{i} \leq 2 \varepsilon
$$

for all $z$ in $\mathbb{R}^{3}$. Suppose $y=(y(1), y(2), \ldots)$ is in $X$ such that $\left\|x_{n} \pm y\right\| \leq 1+\delta$. Then

$$
\left\|e_{1} \pm y(n)\right\| \|_{n} \leq 1+\delta \text { and }\left\|e_{0} \pm y(i)\right\|_{i} \leq 1+\delta
$$

for all $i \neq n$ in $\mathbb{N}$, and hence

$$
\|y(n) \mid\|_{n} \leq \varepsilon \text { and }\|y(i)\|_{i} \leq 2 \varepsilon
$$

for all $i \neq n$ in $\mathbb{N}$. Thus $\|y\| \leq 2 \varepsilon$. This shows that $x_{n}$ is a strongly extreme point of $B_{X}$ and Claim 1 is established.

Claim 2. The sequence $\left\{x_{n}\right\}_{n=1}^{\infty}$ converges weakly to $x_{0}$ in $X$. 
To establish this claim, note that

$$
x_{n}-x_{0}=(\underbrace{0,0, \ldots, 0}_{n-1}, e_{1}-e_{0}, 0,0, \ldots)
$$

for each $n$ in $\mathbb{N}$ and hence the sequence $\left\{x_{n}-x_{0}\right\}_{n=1}^{\infty}$ is actually in $c_{0}\left(X_{i}\right)$. Recall that the dual of $c_{0}\left(X_{i}\right)$ is $\ell^{1}\left(X_{i}^{*}\right)$ and so if $x^{*}=\left(x^{*}(1), x^{*}(2), \ldots\right)$ is in $\ell^{1}\left(X_{i}^{*}\right)$, then

$$
\left|\left\langle x^{*}, x_{n}-x_{0}\right\rangle\right|=\left|\left\langle x^{*}(n), e_{1}-e_{0}\right\rangle\right| \leq 2\left|\left\|x^{*}(n) \mid\right\|_{n}\right.
$$

for each $n$ in $\mathbb{N}$. But $\left.\lim _{n \rightarrow \infty}\left|\| x^{*}(n)\right|\right|_{n}=0$ since $\left\|x^{*}\right\|=\sum_{n=1}^{\infty}\left\|\left|x^{*}(n) \|\right|_{n}\right.$. This shows that $\left\{x_{n}-x_{0}\right\}_{n=1}^{\infty}$ converges weakly to 0 in $c_{0}\left(X_{i}\right)$ and hence converges weakly to 0 in $X=\ell^{\infty}\left(X_{i}\right)$. Thus Claim 2 is established.

Let $K=\left\{\frac{1}{n}: n \in \mathbb{N}\right\} \cup\{0\}$ with the topology inherited from $\mathbb{R}$, and then define $f: K \rightarrow X$ by $f\left(\frac{1}{n}\right)=x_{n}$, for each $n$ in $\mathbb{N}$, and $f(0)=x_{0}$. Then $f$ is in $C(K,(X, w))$ by Claim 2 , and note $\|f\|=1$. Let $D=\left\{\frac{1}{n}: n \in \mathbb{N}\right\}$ and observe that $D$ is the only proper, dense subset of $K$ and that $f(D)$ is not a uniformly strongly extreme subset of $B_{X}$; this last observation follows by examining the point $e_{1}$ in the unit ball $B_{X_{i}}$ as $i$ increases. It follows, by Theorem 5 , that $f$ is not a strongly extreme point of $B_{C(K,(X, w))}$; yet, by Claim 1, every value of $f$ is a strongly extreme point of $B_{X}$. This completes Example 7 .

As mentioned above, the last two examples show that the strongly extreme point situation in the unit ball of $C(K,(X, w))$ is quite different from that in the unit ball $C(K, X)$ when viewed from the perspective of an element $f$ being strongly extreme point valued at every $t$ in $K$. However, from the perspective of an element $f$ being uniformly strongly extreme point valued on a dense subset of $K$, the situations in $C(K,(X, w))$ and $C(K, X)$ are exactly the same, as is seen from Theorem 5 and part (iv) of the following proposition (which may be read with or without the parenthetical "and $G_{\delta}$ ").

Proposition 8. Let $f$ be an element of $C(K, X)$. The following assertions are equivalent:

(i) $f$ is a strongly extreme point of $B_{C(K, X)}$.

(ii) $f(t)$ is a strongly extreme point of $B_{X}$ for each $t$ in $K$.

(iii) $f(K)$ is a uniformly strongly extreme subset of $B_{X}$.

(iv) there exists a dense (and $G_{\delta}$ ) subset $D$ of $K$ such that $f(D)$ is a uniformly strongly extreme subset of $B_{X}$.

Proof. The equivalence of (i) and (ii) is from [DHS]. Suppose (ii) is true for $f$ in $C(K, X)$. If (iii) fails for $f$, then, following a line of argument from [DHS], there exist $\varepsilon>0$ and sequences $\left\{t_{n}\right\}$ in $K$ and $\left\{z_{n}\right\}$ in $X$ such that, for all $n$ in $\mathbb{N}$,

$$
\left\|f\left(t_{n}\right) \pm z_{n}\right\| \leq 1+\frac{1}{n} \text { and }\left\|z_{n}\right\|>\varepsilon
$$

Since $K$ is compact, there exist a subnet $\left\{t_{n_{\lambda}}\right\}$ of $\left\{t_{n}\right\}$ and $t_{0}$ in $K$ such that $\lim _{\lambda} t_{n_{\lambda}}=t_{0}$. Since $f\left(t_{0}\right)$ is a strongly extreme point of $B_{X}$, there exists $n_{0}$ in $\mathbb{N}$ such that $\left\|f\left(t_{0}\right) \pm z\right\| \leq 1+\frac{1}{n_{0}}$ for $z$ in $X$; then $\|z\| \leq \varepsilon$. Using the continuity of $f$ at $t_{0}$, choose $\lambda_{0}$ such that

$$
\left\|f\left(t_{n_{\lambda_{0}}}\right)-f\left(t_{0}\right)\right\|<\frac{1}{2 n_{0}} \quad \text { and } \quad n_{\lambda_{0}}>2 n_{0} .
$$


Then

$$
\begin{aligned}
\left\|f\left(t_{0}\right) \pm z_{n_{\lambda_{0}}}\right\| & \leq\left\|f\left(t_{0}\right)-f\left(t_{n_{\lambda_{0}}}\right)\right\|+\left\|f\left(t_{n_{\lambda_{0}}}\right) \pm z_{n_{\lambda_{0}}}\right\| \\
& <\frac{1}{2 n_{0}}+1+\frac{1}{n_{\lambda_{0}}} \\
& <1+\frac{1}{n_{0}}
\end{aligned}
$$

yet $\left\|z_{n_{\lambda_{0}}}\right\|>\varepsilon$, contrary to the choice of $n_{0}$. This shows that (ii) implies (iii). That (iii) implies (iv) is immediate. Finally, if (iv) is true for $f$ in $C(K, X)$, then Theorem 5 yields that $f$ is a strongly extreme point of $B_{C(K,(X, w))}$, and hence, since $C(K, X)$ is a subspace of $C(K,(X, w))$, assertion (i) follows. This completes the proof.

An immediate corollary of Theorem 5 and Proposition 8 is formally stated below. Although the corresponding statement for extreme points is unlikely to hold, the authors know of no counterexample.

Corollary 9. If $f$ is a strongly extreme point of the unit ball of $C(K, X)$, then $f$ is also a strongly extreme point of the unit ball of $C(K,(X, w))$.

As an immediate consequence of the next theorem, it follows that the unit ball of $C(K,(X, w))$ contains neither denting points nor strongly exposed points.

Theorem 10. The unit ball of $C(K,(X, w))$ contains no point of sequential continuity.

Proof. Let $f$ be in $B_{C(K,(X, w))}$ and set $D=\{t \in K: f$ is norm continuous at $t\}$. Recall that $D$ is dense in $K$. Since $K$ is infinite, there exists a pairwise disjoint sequence $\left\{V_{n}\right\}_{n=1}^{\infty}$ of nonempty, open subsets of $K$. For each $n$ in $\mathbb{N}$, choose a point $t_{n}$ in $V_{n} \cap D$ and use the norm continuity of $f$ at $t_{n}$ to obtain an open subset $U_{n}$ containing $t_{n}$ such that $U_{n} \subseteq V_{n}$ and $\operatorname{diam} f\left(U_{n}\right)<\frac{1}{6}$. Since $\left\|f\left(t_{n}\right)\right\| \leq 1$ for each $n$ in $\mathbb{N}$, choose $x_{n}$ in $X$ such that $\left\|x_{n}\right\|=\frac{1}{6}$ and $\left\|f\left(t_{n}\right)+x_{n}\right\| \leq \frac{5}{6}$; it follows that, for all $t$ in $U_{n}$,

$$
\begin{aligned}
\left\|f(t)+x_{n}\right\| & \leq\left\|f(t)-f\left(t_{n}\right)\right\|+\left\|f\left(t_{n}\right)+x_{n}\right\| \\
& \leq \frac{1}{6}+\frac{5}{6} \\
& =1 .
\end{aligned}
$$

For each $n$ in $\mathbb{N}$, choose a continuous function $g_{n}: K \rightarrow[0,1] \operatorname{such}$ that $\operatorname{supp}\left(g_{n}\right) \subseteq$ $U_{n}$ and $g_{n}\left(t_{n}\right)=1$, and then define $h_{n}: K \rightarrow X$ by $h_{n}(t)=g_{n}(t) x_{n}$ for $t$ in $K$. Note that $h_{n}$ is in $C(K, X)$ and $\left\|h_{n}\right\|=\frac{1}{6}$ for each $n$ in $\mathbb{N}$.

Claim. The sequence $\left\{h_{n}\right\}_{n=1}^{\infty}$ converges weakly to 0 in $C(K, X)$. 
To establish this claim, let $\mu$ be in $C(K, X)^{*}$. Since $\operatorname{supp}\left(h_{n}\right) \subseteq U_{n}$, it follows that

$$
\begin{aligned}
\left|\left\langle\mu, h_{n}\right\rangle\right| & =\left|\int_{K} h_{n} d \mu\right| \\
& =\left|\int_{U_{n}} g_{n}(t) x_{n} d \mu(t)\right| \\
& \leq \int_{U_{n}}\left|g_{n}(t)\right|\left\|x_{n}\right\| d|\mu|(t) \\
& \leq|\mu|\left(U_{n}\right)
\end{aligned}
$$

for each $n$ in $\mathbb{N}$. Since the sequence $\left\{U_{n}\right\}_{n=1}^{\infty}$ is pairwise disjoint, it follows that $|\mu|\left(U_{n}\right) \rightarrow 0$. This combined with the last inequality establishes the claim.

Now, for each $n$ in $\mathbb{N}$, let $f_{n}=f+h_{n}$. Then the sequence $\left\{f_{n}\right\}_{n=1}^{\infty}$ is in $C(K,(X, w))$. Note that if $t$ is in $K \backslash \operatorname{supp}\left(g_{n}\right)$, then

$$
\left\|f_{n}(t)\right\|=\|f(t)\| \leq 1
$$

and if $t$ is in $\operatorname{supp}\left(g_{n}\right)$, then, since $t$ is in $U_{n}$,

$$
\begin{aligned}
\left\|f_{n}(t)\right\| & =\left\|f(t)+h_{n}(t)\right\| \\
& \leq\left(1-g_{n}(t)\right)\|f(t)\|+g_{n}(t)\left\|f(t)+x_{n}\right\| \\
& \leq\left(1-g_{n}(t)\right)+g_{n}(t) \\
& =1 .
\end{aligned}
$$

Thus $\left\{f_{n}\right\}_{n=1}^{\infty}$ is in $B_{C(K,(X, w))}$. Finally, note that $\left\{f_{n}\right\}_{n=1}^{\infty}$ converges weakly to $f$ by the claim above, but $\left\{f_{n}\right\}$ does not converge in norm to $f$ since $\left\|f_{n}-f\right\|=$ $\left\|h_{n}\right\|=\frac{1}{6}$ for each $n$ in $\mathbb{N}$. This shows that $f$ is not a point of sequential continuity of $B_{C(K,(X, w))}$, and the proof is complete.

$$
\text { THe SPACE } C(K,(X, w))^{*}
$$

The absence of a concrete representation of the space $C(K,(X, w))^{*}$ makes an investigation of the extremal structure of its unit ball difficult. From [ADLR], it is known that $C(K, X)^{*}$ is a complemented subspace of $C(K,(X, w))^{*}$ for any Banach space $X$ and any compact Hausdorff space $K$. Indeed,

$$
C(K,(X, w))^{*}=C(K, X)^{*} \oplus C(K, X)^{\perp} .
$$

Thus an extreme point of $B_{C(K, X)^{*}}$ has a chance to be an extreme point of

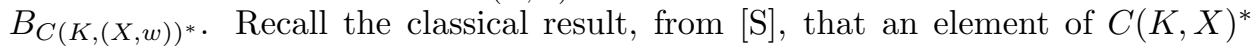
is an extreme point of $B_{C(K, X)^{*}}$ if and only if it has the form $L_{k, x^{*}}$ where $k$ is in $K$ and $x^{*}$ is an extreme point of $B_{X^{*}}$, with the action of this functional on an $f$ in $C(K, X)$ given by

$$
\left\langle L_{k, x^{*}}, f\right\rangle=\left\langle x^{*}, f(k)\right\rangle .
$$

So one question is: for which (if any) extreme points $x^{*}$ of $B_{X^{*}}$, are the functionals $L_{k, x^{*}}$ extreme points of $B_{C(K,(X, w))^{*}}$ ? From [ADLR], every extreme point $F^{*}$ of $B_{C(K,(X, w))^{*}}$ is a so-called point functional, that is, there exists a point $y$ in $Y$, the Stone-Čech compactification of $K \times B_{X^{*}}$, such that $F^{*}=\left.\delta_{y}\right|_{C(K,(X, w))}$, where $\delta_{y}$ denotes the functional in $C(Y)^{*}$ given by point mass measure at $y$. Here $C(K,(X, w))$ is considered to be a subspace of $C(Y)$ as described in the Definitions and Preliminaries section. Note that if $y=\left(k, x^{*}\right)$ is in $K \times B_{X^{*}}$, then 
$\left.\delta_{y}\right|_{C(K,(X, w))}=L_{k, x^{*}}$. Also, from [ADLR], every point functional is supported at a point in $K$, that is, for $y$ in $Y$ there is a point $k$ in $K$, an element $x^{*}$ in $X^{*}$ and $F^{\perp}$ in $C(K, X)^{\perp}$ such that

$$
\left.\delta_{y}\right|_{C(K,(X, w))}=L_{k, x^{*}}+F^{\perp}
$$

thus every extreme point $F^{*}$ of $B_{C(K,(X, w))^{*}}$ can be written in the form $F^{*}=$ $L_{k, x^{*}}+F^{\perp}$. So another question is: are there extreme points of $B_{C(K,(X, w))^{*}}$ where $F^{\perp}$ is nonzero?

With regard to the first question, from $[\mathrm{C}]$, the functional $L_{k, x^{*}}$ need not be an extreme point of $B_{C(K,(X, w))^{*}}$ whenever $x^{*}$ is an extreme point of $B_{X^{*}}$. This is in marked contrast to the situation in $C(K, X)^{*}$ as given above. However, from [L] as illuminated in [C], if $x^{*}$ is a weak ${ }^{*}$ strongly exposed point of $B_{X^{*}}$ and $k$ is in $K$, then $L_{k, x^{*}}$ is an extreme point of $B_{C(K,(X, w))^{*}}$. The results given below, Theorem 14 and Theorem 16, are considerable strengthenings of this last statement. Indeed, if $x^{*}$ is a weak ${ }^{*}$ strongly exposed point of $B_{X^{*}}$ and $k$ is in $K$, then (Theorem 14)

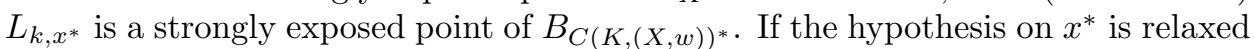
to that of being a weak* denting point of $B_{X^{*}}$, then it follows (Theorem 16) that $L_{k, x^{*}}$ is a denting point of $B_{C(K,(X, w))^{*}}$; in particular, $L_{k, x^{*}}$ is a point of continuity of $B_{C(K,(X, w))^{*}}$. In both of these results the conclusion for $L_{k, x^{*}}$ is much stronger than that of just being an extreme point of $B_{C(K,(X, w))^{*}}$, but the hypothesis on $x^{*}$ is also much stronger than that of just being an extreme pont of $B_{X^{*}}$. There are some situations, as illustrated by Proposition 11 below, in which $L_{k, x^{*}}$ is an extreme point of $B_{C(K,(X, w))^{*}}$ whenever $x^{*}$ is an extreme point of $B_{X^{*}}$. The proof of Proposition 11 is straightforward since under its hypothesis the sum in $(*)$ is an $\ell^{1}$-sum and so the classical result from [S], mentioned above, can be applied in the case of an extreme point, a result from [HS] can be applied in the case of a strongly extreme point, and results from [RS] can be applied in the cases of a denting point or a strongly exposed point.

Proposition 11. Let $X$ be a Banach space such that $C(K, X)$ is an $M$-ideal in $C(K,(X, w))$. Then $L_{k, x^{*}}$ is an extreme point (respectively, strongly extreme point, denting point, strongly exposed point) of $B_{C(K,(X, w))^{*}}$ whenever $k$ is in $K$ and $x^{*}$ is an extreme point (respectively, strongly extreme point, denting point, strongly exposed point) of $B_{X^{*}}$.

It should be noted, from [ADLR], that $C(K, X)$ is an M-ideal in $C(K,(X, w))$ whenever $X$ has the Schur approximation property. Also, from [ADLR], the space $c_{0}$, the space $c_{0}(\Gamma)$ and $c_{0}$-sums of spaces with the Schur property have the Schur approximation property.

With regard to the second question, there are spaces $X$ for which there are extreme points of $B_{C(K,(X, w))^{*}}$ other than those of the form $L_{k, x^{*}}$. For example, let $X=c_{0}$. Then the sum in $(*)$ is an $\ell^{1}$-sum and so any extreme point of $B_{C(K, X)^{\perp}}$

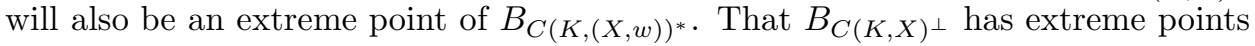
follows by the Krein-Milman theorem since $B_{C(K, X) \perp}$ is weak* compact and convex.

Since the next two theorems involve the functional $L_{k, x^{*}}$ for $x^{*}$ in $B_{X^{*}}$ with $\left\|x^{*}\right\|$ $=1$, in the proofs $Y$ will denote the Stone-Čech compactification of $K \times S_{X^{*}}$ and, in a manner completely analogous to that given in the Definitions and Preliminaries section (just replace $B_{X^{*}}$ by $\left.S_{X^{*}}\right)$, the space $C(K,(X, w))$ will be considered as a 
subspace of $C(Y)$. Also, for $\varepsilon>0$, let

$$
S_{\varepsilon}^{+}=\left\{z^{*} \in S_{X^{*}}:\left\|z^{*}-x^{*}\right\|<\varepsilon\right\}
$$

and

$$
S_{\varepsilon}^{-}=\left\{z^{*} \in S_{X^{*}}:\left\|z^{*}-\left(-x^{*}\right)\right\|<\varepsilon\right\} ;
$$

and then let $S_{\varepsilon}=S_{\varepsilon}^{+} \cup S_{\varepsilon}^{-}$and $S_{\varepsilon}^{c}=S_{X^{*}} \backslash S_{\varepsilon}$.

The proofs of Theorem 14 and Theorem 16 require a great deal of work but the rewards are great as well since, for the first time, some strongly exposed points and some denting points of the unit ball of $C(K,(X, w))^{*}$ are identified without restriction on $X$ or $K$. The proofs of both theorems will be facilitated by the following two technical results.

Lemma 12. Let $\varepsilon$ and $\delta$ be positive numbers each less than $\frac{1}{2}$ and let $x$ in $S_{X}$ and $x^{*}$ in $S_{X^{*}}$ be such that $\left\langle x^{*}, x\right\rangle>1-\delta$ and $\operatorname{diam} S\left(x, B_{X^{*}}, \delta\right)<\varepsilon$. Let $k$ be a point of $K$, let $V$ be an open subset of $K$ that contains $k$ and let $g: K \rightarrow[0,1]$ be a continuous function such that $g(k)=1$ and $\operatorname{supp}(g) \subseteq V$. Let $Y$ be the Stone- $\breve{C e c h}$ compactification of $K \times S_{X^{*}}$. If $\mu$ in $C(Y)^{*}$ is such that $\|\mu\| \leq 1$ and

$$
\int_{Y} \overline{g \otimes x} d \mu>1-\delta \varepsilon
$$

then

$$
\mu^{+}\left(\overline{V \times S_{\varepsilon}^{+}}\right)+\mu^{-}\left(\overline{V \times S_{\varepsilon}^{-}}\right)>1-\varepsilon .
$$

Proof. If $z^{*}$ is in $S_{\varepsilon}^{+}$, then $\left\|z^{*}-x^{*}\right\|<\varepsilon$. This combined with $\left\langle x^{*}, x\right\rangle>1-\delta$ and $\varepsilon+\delta<1$ yields that $\left\langle z^{*}, x\right\rangle \geq 0$. Thus $g \otimes x \geq 0$ on $V \times S_{\varepsilon}^{+}$, and hence it follows that $\overline{g \otimes x} \geq 0$ on $\overline{V \times S_{\varepsilon}^{+}}$, where the closure is in $Y$. Similarly, it can be shown that $\overline{g \otimes x} \leq 0$ on $\overline{V \times S_{\varepsilon}^{-}}$. Thus

$$
\int_{\overline{V \times S_{\varepsilon}^{+}}} \overline{g \otimes x} d \mu^{-} \geq 0 \text { and } \int_{\overline{V \times S_{\varepsilon}^{-}}} \overline{g \otimes x} d \mu^{+} \leq 0 .
$$

From these inequalities and the fact that $\|\overline{g \otimes x}\| \leq 1$, it follows that

$$
\begin{aligned}
\int_{\overline{V \times S_{\varepsilon}}} \overline{g \otimes x} d \mu=\int_{\overline{V \times S_{\varepsilon}^{+}}} \overline{g \otimes x} d \mu^{+}-\int_{\overline{V \times S_{\varepsilon}^{+}}} \overline{g \otimes x} d \mu^{-} \\
+\int_{\overline{V \times S_{\varepsilon}^{-}}} \overline{g \otimes x} d \mu^{+}-\int_{\overline{V \times S_{\varepsilon}^{-}}} \overline{g \otimes x} d \mu^{-}
\end{aligned}
$$

$$
\begin{aligned}
& \leq \int_{\overline{V \times S_{\varepsilon}^{+}}} \overline{g \otimes x} d \mu^{+}-\int_{\overline{V \times S_{\varepsilon}^{-}}} \overline{g \otimes x} d \mu^{-} \\
& \leq \mu^{+}\left(\overline{V \times S_{\varepsilon}^{+}}\right)+\mu^{-}\left(\overline{V \times S_{\varepsilon}^{-}}\right) .
\end{aligned}
$$

Now, for $z^{*}$ in $S_{\varepsilon}^{c}$, it is the case that

$$
\left\|z^{*}-x^{*}\right\| \geq \varepsilon \text { and }\left\|z^{*}-\left(-x^{*}\right)\right\| \geq \varepsilon,
$$


and hence, since $\operatorname{diam} S\left(x, B_{X^{*}}, \delta\right)<\varepsilon$, it follows that $z^{*}$ is in neither $S\left(x, B_{X^{*}}, \delta\right)$ nor $S\left(-x, B_{X^{*}}, \delta\right)$. This yields $\left\langle z^{*}, x\right\rangle \leq 1-\delta$ and $\left\langle z^{*},-x\right\rangle \leq 1-\delta$, that is, $\left|\left\langle z^{*}, x\right\rangle\right| \leq 1-\delta$. Thus, for $\left(t, z^{*}\right)$ in $V \times S_{\varepsilon}^{c}$,

$$
\left|(g \otimes x)\left(t, z^{*}\right)\right| \leq\left|\left\langle z^{*}, x\right\rangle\right| \leq 1-\delta,
$$

and hence $|\overline{g \otimes x}| \leq 1-\delta$ on $\overline{V \times S_{\varepsilon}^{c}}$. Also, $\overline{g \otimes x}=0$ on $\overline{V^{c} \times S_{X^{*}}} \operatorname{since} \operatorname{supp}(g) \subseteq$ $V$. Now it follows that

$$
\int_{Y \backslash \overline{V \times S_{\varepsilon}}} \overline{g \otimes x} d \mu \leq(1-\delta)|\mu|\left(Y \backslash \overline{V \times S_{\varepsilon}}\right) .
$$

Combining (1) and (2) yields

$$
\begin{aligned}
1-\delta \varepsilon< & \int_{Y} \overline{g \otimes x} d \mu \\
& =\int_{\overline{V \times S_{\varepsilon}}} \overline{g \otimes x} d \mu+\int_{Y \backslash \overline{V \times S_{\varepsilon}}} \overline{g \otimes x} d \mu \\
\leq & \mu^{+}\left(\overline{V \times S_{\varepsilon}^{+}}\right)+\mu^{-}\left(\overline{V \times S_{\varepsilon}^{-}}\right) \\
& \quad+(1-\delta)|\mu|\left(Y \backslash \overline{V \times S_{\varepsilon}}\right) \\
\leq & |\mu|(Y)-\delta|\mu|\left(Y \backslash \overline{V \times S_{\varepsilon}}\right) .
\end{aligned}
$$

From this and the fact that $\|\mu\| \leq 1$, it follows that $|\mu|\left(Y \backslash \overline{V \times S_{\varepsilon}}\right)<\varepsilon$. Now, using this and the first two inequalities listed in (3) yields

$$
\begin{aligned}
\mu^{+}\left(\overline{V \times S_{\varepsilon}^{+}}\right)+\mu^{-}\left(\overline{V \times S_{\varepsilon}^{-}}\right) & \geq 1-\delta \varepsilon-(1-\delta)|\mu|\left(Y \backslash \overline{V \times S_{\varepsilon}}\right) \\
& >1-\delta \varepsilon-(1-\delta) \varepsilon \\
& =1-\varepsilon .
\end{aligned}
$$

This completes the proof.

Lemma 13. Let $\varepsilon$ and $\delta$ be positive numbers each less than $\frac{1}{2}$ and let $x$ in $S_{X}$ and $x^{*}$ in $S_{X^{*}}$ be such that $\left\langle x^{*}, x\right\rangle>1-\delta \varepsilon$ and $\operatorname{diam} S\left(x, B_{X^{*}}, \delta\right)<\varepsilon$. Let $k$ be in $K$ and let $N(k)$ denote the collection of all open subsets of $K$ which contain the point $k$. For each $V$ in $N(k)$, choose a continuous function $g_{V}: K \rightarrow[0,1]$ such that $g_{V}(k)=1$ and $\operatorname{supp}\left(g_{V}\right) \subseteq V$. Then $\left\{g_{V} \otimes x\right\}_{V \in N(k)}$ forms a net (ordered by inclusion) in $B_{C(K,(X, w))}$ which can be considered as a subset of $B_{C(K,(X, w))^{* *} \text {. Let }}$ $T$ be a weak* cluster point of this net. Then

(i) $\left\langle T, L_{k, x^{*}}\right\rangle=\left\langle x^{*}, x\right\rangle>1-\delta \varepsilon$ and $\|T\| \leq 1$,

(ii) if $\left\langle T, F^{*}\right\rangle>1-\delta \varepsilon$, where $F^{*}$ is in $B_{C(K,(X, w))^{*}}$, it follows that $\left\|F^{*}-L_{k, x^{*}}\right\| \leq$ $4 \varepsilon$.

Proof. Note that since

$$
\left\langle L_{k, x^{*}}, g_{V} \otimes x\right\rangle=\left\langle x^{*}, x\right\rangle>1-\delta \varepsilon
$$

for every $V$ in $N(k)$, it follows that $\left\langle T, L_{k, x^{*}}\right\rangle=\left\langle x^{*}, x\right\rangle>1-\delta \varepsilon$. It is clear that $\|T\| \leq 1$. Suppose $F^{*}$ is in $B_{C(K,(X, w))^{*}}$ such that $\left\langle T, F^{*}\right\rangle>1-\delta \varepsilon$. Let $Y$ be the Stone-Čech compactification of $K \times S_{X^{*}}$ and consider $C(K,(X, w))$ as 
a subspace of $C(Y)$. Apply the Hahn-Banach theorem to obtain $\mu$ in $C(Y)^{*}$ such that $\mu$ extends $F^{*}$ while $\|\mu\|=\left\|F^{*}\right\|$; thus

$$
\left\langle F^{*}, f\right\rangle=\int_{Y} \bar{f} d \mu \text { for all } f \text { in } C(K,(X, w)) .
$$

Claim. For every $U$ in $N(k)$,

$$
\mu^{+}\left(\overline{U \times S_{\varepsilon}^{+}}\right)+\mu^{-}\left(\overline{U \times S_{\varepsilon}^{-}}\right)>1-\varepsilon .
$$

Since $T$ is a weak* cluster point of $\left\{g_{V} \otimes x\right\}_{V \in N(k)}$ in $C(K,(X, w))^{* *}$, from the hypothesis on $F^{*}$, there exists $V$ in $N(k)$ such that $V \subset U$ and $\left\langle F^{*}, g_{V} \otimes x\right\rangle>$ $1-\delta \varepsilon$. Hence, by (1),

$$
\int_{Y} \overline{g_{V} \otimes x} d \mu>1-\delta \varepsilon
$$

Lemma 12 is applicable, and it yields that

$$
\begin{aligned}
\mu^{+}\left(\overline{U \times S_{\varepsilon}^{+}}\right)+\mu^{-}\left(\overline{U \times S_{\varepsilon}^{-}}\right) & \geq \mu^{+}\left(\overline{V \times S_{\varepsilon}^{+}}\right)+\mu^{-}\left(\overline{V \times S_{\varepsilon}^{-}}\right) \\
& >1-\varepsilon
\end{aligned}
$$

This establishes the Claim.

Let $f$ be any element of $B_{C(K,(X, w))}$. By the weak continuity of $f$ at $k$, there exists $V$ in $N(k)$ such that

$$
\left|\left\langle x^{*}, f(t)-f(k)\right\rangle\right|<\varepsilon \text { for all } t \text { in } V .
$$

If $\left(t, z^{*}\right)$ is in $V \times S_{\varepsilon}^{+}$, then $\left\|z^{*}-x^{*}\right\|<\varepsilon$ and hence, by (2),

$$
\begin{aligned}
\left|\tilde{f}\left(t, z^{*}\right)-\left\langle x^{*}, f(k)\right\rangle\right| & =\left|\left\langle z^{*}, f(t)\right\rangle-\left\langle x^{*}, f(k)\right\rangle\right| \\
& \leq\left|\left\langle z^{*}-x^{*}, f(t)\right\rangle\right|+\left|\left\langle x^{*}, f(t)-f(k)\right\rangle\right| \\
& <\varepsilon+\varepsilon .
\end{aligned}
$$

This yields that

$$
\left|\bar{f}-\left\langle x^{*}, f(k)\right\rangle\right| \leq 2 \varepsilon \text { on } \overline{V \times S_{\varepsilon}^{+}}
$$

Similarly, it can be shown that

$$
\left|\bar{f}+\left\langle x^{*}, f(k)\right\rangle\right| \leq 2 \varepsilon \text { on } \overline{V \times S_{\varepsilon}^{-}}
$$

From the Claim and the fact that $\|\mu\|=|\mu|(Y) \leq 1$, it follows that

$$
\mu^{+}\left(\overline{V \times S_{\varepsilon}^{-}}\right)+\mu^{-}\left(\overline{V \times S_{\varepsilon}^{+}}\right)+|\mu|\left(Y \backslash \overline{V \times S_{\varepsilon}}\right)<\varepsilon .
$$


Combining this with (1), (3), (4), the Claim, and the fact that $\|f\| \leq 1$, it follows that

$$
\begin{aligned}
\mid\left\langle F^{*}-\right. & \left.L_{k, x^{*}}, f\right\rangle \mid \\
= & \left|\int_{Y} \bar{f} d \mu-\left\langle x^{*}, f(k)\right\rangle\right| \\
\leq & \left|\int_{V \times S_{\varepsilon}^{+}} \bar{f} d \mu^{+}-\int_{\overline{V \times S_{\varepsilon}^{-}}} \bar{f} d \mu^{-}-\left\langle x^{*}, f(k)\right\rangle\right| \\
& +\left|\int_{\overline{V \times S_{\varepsilon}^{-}}} \bar{f} d \mu^{+}-\int_{\overline{V \times S_{\varepsilon}^{+}}} \bar{f} d \mu^{-}+\int_{Y \backslash \overline{V \times S_{\varepsilon}}} \bar{f} d \mu\right| \\
\leq & \mid \int_{\overline{V \times S_{\varepsilon}^{+}}}\left(\bar{f}-\left\langle x^{*}, f(k)\right\rangle\right) d \mu^{+}-\int_{\overline{V \times S_{\varepsilon}^{-}}}\left(\bar{f}+\left\langle x^{*}, f(k)\right\rangle\right) d \mu^{-} \\
& +\mu^{+}\left(\overline{V \times S_{\varepsilon}^{-}}\right)+\mu^{-}\left(\overline{V \times S_{\varepsilon}^{+}}\right)+|\mu|\left(Y \backslash \overline{V \times S_{\varepsilon}}\right) \\
\leq & 2 \varepsilon \mu^{+}\left(\overline{V \times S_{\varepsilon}^{+}}\right)+2 \varepsilon \mu^{-}\left(\overline{V \times S_{\varepsilon}^{-}}\right) \\
& \left.\left.+\mid 1-\mu^{+}\right)\right] \mid \\
& +\varepsilon \\
< & 2 \varepsilon|\mu|\left(\overline{V \times S_{\varepsilon}}\right)+\varepsilon+\varepsilon \\
\leq & 4 \varepsilon .
\end{aligned}
$$

Since $f$ was arbitrary, it follows that $\left\|F^{*}-L_{k, x^{*}}\right\| \leq 4 \varepsilon$. This completes the proof.

Theorem 14. If $k$ is in $K$ and $x^{*}$ is a weak ${ }^{*}$ strongly exposed point of $B_{X^{*}}$, then $L_{k, x^{*}}$ is a strongly exposed point of $B_{C(K,(X, w))^{*}}$.

Proof. Let $x$ in $S_{X}$ be such that $x$ weak* strongly exposes $B_{X^{*}}$ at $x^{*}$. Let $N(k)$ denote the collection of all open subsets of $K$ which contain the point $k$. For each $V$ in $N(k)$, choose a continuous function $g_{V}: K \rightarrow[0,1]$ such that $g_{V}(k)=1$ and $\operatorname{supp}\left(g_{V}\right) \subseteq V$. Then $\left\{g_{V} \otimes x\right\}_{V \in N(k)}$ forms a net (ordered by inclusion) in $B_{C(K,(X, w))}$ which can be considered as a subset of $B_{C(K,(X, w))^{* *} \text {. Let } T \text { be a weak* }}$ cluster point of this net. It will be shown that $T$ strongly exposes $B_{C(K,(X, w))^{*}}$ at $L_{k, x^{*}}$. Lemma 13 yields that $\left\langle T, L_{k, x^{*}}\right\rangle=\left\langle x^{*}, x\right\rangle=1$ and $\|T\| \leq 1$; hence $\|T\|=1$. Suppose $\left\{F_{n}^{*}\right\}_{n=1}^{\infty}$ is a sequence in $B_{C(K,(X, w))^{*}}$ such that $\lim _{n \rightarrow \infty}\left\langle T, F_{n}^{*}\right\rangle=1$; the proof will be complete once it is shown that $\lim _{n \rightarrow \infty}\left\|F_{n}^{*}-L_{k, x^{*}}\right\|=0$. Let $\varepsilon$ be such that $0<\varepsilon<\frac{1}{2}$. Choose $\delta$ such that $0<\delta<\frac{1}{2}$ and $\operatorname{diam} S\left(x, B_{X^{*}}, \delta\right)<\varepsilon$. Since $\lim _{n \rightarrow \infty}\left\langle T, F_{n}^{*}\right\rangle=1$, Lemma 13 (ii) applies and yields that $\left\|F_{n}^{*}-L_{k, x^{*}}\right\| \leq 4 \varepsilon$ for all sufficiently large $n$ in $\mathbb{N}$. This completes the proof.

The proof of the following corollary is immediate, since its hypothesis implies that every $x^{*}$ in $S_{X^{*}}$ is a weak ${ }^{*}$ strongly exposed point of $B_{X^{*}}$.

Corollary 15. If $X$ is a reflexive Banach space with Fréchet differentiable norm (for example, if $X$ is any of the spaces $\ell^{p}(\Gamma)$ or $L^{p}(\mu)$ for $1<p<\infty$ ), then $L_{k, x^{*}}$ is a strongly exposed point of $B_{C(K,(X, w))^{*}}$ whenever $k$ is in $K$ and $x^{*}$ is in $S_{X^{*}}$. 
The next result should be compared with Theorem 14; it applies to a wider variety of extreme points but, as expected, delivers a little less in its conclusion. Nevertheless, it does yield denting points of $B_{C(K,(X, w)) *}$ with no restrictions on $K$ or $X$.

Theorem 16. If $k$ is in $K$ and $x^{*}$ is a weak* denting point of $B_{X^{*}}$, then $L_{k, x^{*}}$ is a denting point of $B_{C(K,(X, w))^{*} \text {. }}$

Proof. Let $k$ and $x^{*}$ be as in the hypothesis. Let $\varepsilon$ be such that $0<\varepsilon<\frac{1}{2}$. By Lemma 5 in [HL], there exist $x$ in $S_{X}$ and $\delta>0$ such that

$$
\left\langle x^{*}, x\right\rangle>1-\delta \varepsilon \quad \text { and } \operatorname{diam} S\left(x, B_{X^{*}}, \delta\right)<\varepsilon .
$$

Note that $\delta \leq \varepsilon<\frac{1}{2}$. Now, let $T$ be a weak* cluster point of the net considered in the statement of Lemma 13. Let $\delta_{1}=\|T\|-(1-\delta \varepsilon)$. By Lemma 13 (i), it follows that $\delta_{1}>0$ and $L_{k, x^{*}}$ is in $S\left(T, B_{C(K,(X, w))^{*}}, \delta_{1}\right)$. Let $F^{*}$ be an element of

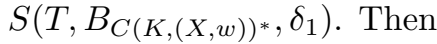

$$
\left\langle T, F^{*}\right\rangle>\|T\|-\delta_{1}=1-\delta \varepsilon
$$

and hence, by Lemma 13 (ii),

$$
\left\|F^{*}-L_{k, x^{*}}\right\| \leq 4 \varepsilon .
$$

Thus

$$
\operatorname{diam} S\left(T, B_{C(K,(X, w))^{*}}, \delta_{1}\right) \leq 8 \varepsilon
$$

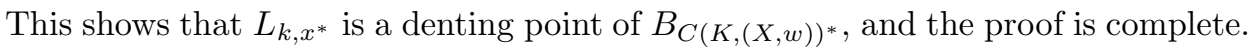

\section{REFERENCES}

[ADLR] J. Arias de Reyna, J. Diestel, V. Lomonosov, and L. Rodrigues-Piazza, Some observations about the space of weakly continuous functions from a compact space into a Banach space, Quaestiones Mathematicae 15 (1992), 415-425. MR 94b:46055

[BLP] R.M. Blumenthal, J. Lindenstrauss and R.R. Phelps, Extreme operators into $C(K)$, Pacific J. Math. 15 (1965), 747-756. MR 35:758

[C] M. Cambern, Some extremum problems for spaces of weakly continuous functions, Function Spaces, Lecture Notes in Pure and Applied Math. 136 (Marcel Dekker 1992), 61-65. MR 93c: 46056

[DD] P. Domanski and L. Drewnowski, The uncomplementability of the space of continuous functions in the space of weakly-continuous functions, Studia Math. 97 (1991), 245-251. MR 92b:46051

[DHS] P.N. Dowling, Z. Hu and M.A. Smith, Extremal structure of the unit ball of $C(K, X)$, Contemp. Math. 144 (1993), 81-85. MR 93j:46042

[G] R. Grząślewicz, Extreme operator-valued continuous maps, Arkiv för Matematik 29 (1991), 73-81. MR 92h:46049

[HL] Z. Hu and B-L Lin, A characterization of weak* denting points in $L^{p}(\mu, X)$, Rocky Mountain J. Math. 24 (1994), 997-1008. MR 96b:46055

[HS] Z. Hu and M.A. Smith, On the extremal structure of the unit ball of the space $C(K, X)^{*}$, Function Spaces, the Second Conference, Lecture Notes in Pure and Applied Math 172 (1995), 205-222. MR 96k:46062

[L] P-K Lin, The isometries of $H^{\infty}(E)$, Pacific J. Math. 143 (1990), 69-77. MR 91f:46075

[LLT] B-L Lin, P-K Lin and S.L. Troyanski, Some geometric and topological properties of the unit sphere in a Banach space, Math. Ann. 274 (1986), 613-616. MR 87k:46036

[M] V. Montesinos, A note on weakly continuous Banach space valued functions, Quaestiones Mathematicae 15 (1992), 501-502. MR 94b:46056 
[RS] W.M. Ruess and C.P. Stegall, Exposed and denting points in duals of operator spaces, Israel J. Math. 53 (1986), 163-190. MR 87j:46015

[S] I. Singer, Linear functionals on the space of continuous mappings of a compact Hausdorff space into a Banach space, Rev. Roumaine Math. Pures Appl. 2 (1957), 301-315. (Russian) MR 20:3445

Division of Mathematics, El Paso Community College, Valle Verde Campus, P.O. Box 20500, El Paso, Texas 79998

Department of Mathematics and Statistics, Miami University, Oxford, Ohio 45056 